\title{
DU FOYER AU FORUM. LA PLACE DES MATRONES ÉQUESTRES DANS LES ACTIVITÉS ÉCONOMIQUES
}

\author{
Anthony Álvarez Melero ${ }^{1}$
}

L'étude de la place des femmes dans l'économie se révèle en général une tâche peu aisée. En effet, à l'instar de bon nombre d'activités impliquant un certain degré d'interventions en public ${ }^{2}$, les Anciens eux-mêmes, par la bouche de Columelle, par exemple, considéraient que ces dames devaient s'en tenir éloignées pour se contenter de la gestion des affaires domestiques $^{3}$. Toutefois, puisque les sources littéraires, où règnent généralement la partialité et les poncifs négatifs à l'égard des femmes, ne nous sont rarement d'un grand secours et semblent même confirmer leur marginalisation, il faut se tourner vers l'épigraphie et la papyrologie pour tenter de trouver une réponse à nos questions.

Dans le cadre de cet exposé, qui ne prétend nullement épuiser un sujet aux vastes et infinies perspectives, en constant renouvellement, je vais m'intéresser plus particulièrement aux parentes de chevaliers. Sans revenir en détail sur l'historiographie de ce groupe «privilégié » de femmes, il me paraît utile de brièvement rappeler que les matrones équestres, telles que je les ai recensées ${ }^{4}$, sont ces filles, petites-filles, épouses, sœurs, mères et aïeules de chevaliers romains attestées dans nos sources, principalement épigraphiques. Cette définition a pu être établie grâce aux prescriptions

1 Investigador Juan de la Cierva, Departamento de Historia antigua, Universidad de Sevilla et SociAAM-Oikoumene, Université libre de Bruxelles. Je tiens à remercier Mmes S. Demougin (EPHE), E. Hemelrijk (UvA), M.-Th. Raepsaet-Charlier (ULB) et M.G. Chic (U. Sevilla) pour leurs relectures, conseils et avis stimulants. Je demeure seul responsable des lacunes et erreurs qui subsistent. Pour les toponymes les plus connus, j'ai opté pour leur dénomination moderne; pour les autres, j'ai conservé la terminologie latine.

2 Pour un aperçu, voir M.-Th. Raepsaet-Charlier, 'Les activités publiques des femmes sénatoriales et équestres sous le Haut-Empire romain', in W. Eck et M. Heil (éd.), Senatores populi Romani. Realität und mediale Präsentation einer Führungsschicht. Kolloquium der Prosopographia Imperii Romani vom 11.-13. Juni 2004 (Stuttgart 2005), 169-212.

3 La lecture de l'introduction au livre 12 de son ouvrage De l'Agriculture est à cet égard éclairante à plus d'un titre: Colum., pr. 12.

4 Il faut savoir que cette appellation n'apparaît que dans le compte-rendu épigraphique des Jeux séculaires de l'an 204 de notre ère pour qualifier les épouses de chevaliers et mères de famille qui prirent part au sellisterne du 2 juin 204: Cf. I.B. Pighi, De ludis saecularibus populi Romani Quiritium libri sex (Amsterdam 1965² [Milan, 1939]) : Fr V/Va 1. 26-29. 
du sénatus-consulte de Larinum, découvert en 1978, daté de l'an 19 de notre ère ${ }^{5}$, et d'un autre sénatus-consulte de $23^{6}$. En dépit de l'importance numérique de ces dames, peu de recherches leur furent consacrées, étant donné la difficulté qu'il y a à les identifier en tant que telles puisqu'elles ne disposaient pas de titre qui leur soit propre. Malgré tout, quelques études ont paru sur la place des femmes dans l'économie ${ }^{7}$, entre autres de l'ordre sénatorial $^{8}$, mais en revanche aucun travail ne s'est exclusivement concentré sur les matrones équestres, si ce n'est en passant, pour les raisons évidentes que je viens d'évoquer'.

Dans les pages qui suivent, seront présentées quelques activités économiques qui témoignent, entre les règnes d'Auguste et de Gallien, de

$5 A E$ 1978, 145.

6 Plin., nat. 33, 32. Ce sénatus-consulte rappelle l'obligation d'ingénuité pour le père, l'aïeul paternel, et le candidat chevalier pour accéder à l'ordre équestre. Afin de déterminer si les épouses, les mères et aïeules des futurs chevaliers étaient soumises à la même contrainte, je les ai comptabilisées dans mon catalogue prosopographique. Sur tout cela, A. Álvarez Melero, 'Matronae equestres ex provincia Lusitania ortae', Revue belge de philologie et d'histoire 86 (2008), 69-95.

7 Que de chemin parcouru en effet depuis l'article pionnier de S. Treggiari, 'Jobs for Women', American Journal of Ancient History 1 (1976), 76-104. A sa suite, sans prétention à l'exhaustivité et en guise d'introduction, ont paru d'autres études telles celles de N. Kampen, Image and Status. Roman Working Women in Ostia (Berlin 1981); J. Maurin, 'Labor matronalis: aspects du travail féminin à Rome', in E. Lévy (éd.), La femme dans les sociétés antiques. Actes des colloques de Strasbourg (mai 1980 et mars 1981) (Strasbourg 1983), 139-155 ; M. Eichenauer, Untersuchungen zur Arbeitswelt der Frauen in der römischen Antike (Francfort 1988); S.R. Joshel, Women, Identity, and Legal Status at Rome. A Study of the Occupational Inscriptions (Norman-Londres 1992); S. Mratschek-Halfmann, Divites et praepotentes. Reichtum und soziale Stellung in der Literatur der Prinzipatszeit (Stuttgart 1993) (Historia Einzelschriften 70), passim; A.M. Andermahr, Totus in praediis. Senatorischer Grundbesitz in Italien in der Frühen und Hohen Kaiserzeit (Bonn 1998), passim; D. Gourévitch et M.-Th. Raepsaet-Charlier, La femme dans la Rome antique (Paris 2001), 183-204; P. Setälä, 'Women and Brick Production. Some New Aspects', in P. Setälä et al. (éd.), Women, Wealth and Power in the Roman Empire (Rome 2002), 181-201 ; C. BriandPonsart, 'Les dames et la terre dans l'Afrique romaine', Histoire et sociétés rurales 19 (2003), 79-9o ; A. Buonopane et F. Cenerini (éd.), Donna e lavoro nella documentazione epigrafica. Atti del I seminario sulla condizione femminile nella documentazione epigrafica (Faenza 2003). Sur l'évergétisme féminin, voir note 11.

8 M.-Th. Raepsaet-Charlier, 'L'activité évergétique des femmes clarissimes sous le HautEmpire', in M.L. Caldelli et al. (éd.), Epigrafia 2006. Atti della XIVe rencontre sur l'épigraphie in onore di Silvio Panciera con altri contributi di colleghi, allievi e collaboratori, III (Rome 2008), 1029-1045.

9 Une exception, toutefois, celle de Raepsaet-Charlier 2005, op. cit. (n. 2), 202-203 au caractère exemplatif mais non exhaustif. Voir aussi J. Andreau, 'Intérêts non agricoles des chevaliers romains ( $\mathrm{II}^{\mathrm{e}}$ siècle av. J.-C.- $\mathrm{III}^{\mathrm{e}}$ siècle ap. J.-C.)', in S. Demougin, H. Devijver et M.-Th. Raepsaet-Charlier (éd.), L'ordre équestre. Histoire d'une aristocratie (II siècle av.J.-C.$I I I^{e}$ siècle ap. J.-C.). Actes du colloque international organisé par Ségolène Demougin, Hubert Devijver et Marie-Thérèse Raepsaet-Charlier (Bruxelles-Leuven, 5-7 octobre 1995) (Rome 1999), 274. 
la richesse dont disposaient des parentes de chevaliers romains, sur la base des dépouillements effectués dans le cadre de ma thèse de doctorat. L'accent sera porté dans un premier temps sur l'évergétisme ainsi que sur les constructions bâties à l'instigation de ces femmes, puis je vais m'interroger sur les sources de leur fortune, afin de déterminer, autant que faire se peut, par quels biais elle était acquise et/ou transmise.

Enfin, avant d'en venir à l'exposé des résultats, il faut signaler que mes recherches ont permis de rassembler un nombre relativement significatif de témoignages. Cependant, dans le cadre de cette communication et pour davantage de maniabilité, je me suis vu contraint de restreindre le champ de mes investigations à un grand ensemble provincial : l'Occident romain. Ce choix s'explique par le volume et la variété des sources ainsi que des cas de figure qu'elles nous présentent. Toutefois, afin de parvenir à des conclusions exhaustives, je vais y inclure quelques données provenant des provinces de l'Orient hellénophone ${ }^{10}$.

Je commencerai par une expression concrète de la richesse de ces femmes, en l'occurrence l'évergétisme. Cette pratique, surgie dans l'Orient hellénophone, héritée de l'époque hellénistique, ayant été amplement et abondamment étudiée par d'illustres devanciers, je m'abstiens donc de répéter ici ce que d'autres ont écrit avant moi ${ }^{11}$. Toutefois, il convient d'en

10 Il est ensuite un cas spécial sur lequel je ne vais pas trop m'appesantir, celui des matronae stolatae qui, à en croire B. Holtheide, furent toutes des parentes de chevaliers et dans le même temps des propriétaires terriennes. Etant donné que ces assertions sont loin d'être avérées, je ne vais prendre en considération que les femmes clairement et indiscutablement apparentées à des chevaliers. Cf. B. Holtheide, 'Matrona stolata - femina stolata', Zeitschrift für Papyrologie und Epigraphik 38 (1980), 127-131.

11 L'ouvrage pionnier est celui de P. Veyne, Le pain et le cirque. Sociologie historique d'un pluralisme politique (Paris 1976). Par la suite, d'autres chercheurs ont complété son travail : E. Melchor Gil, El mecenazgo cívico en la Bética. La contribución de los evergetas al desarrollo de la vida municipal (Cordoue 1994) et Id., La munificiencia cívica en el mundo romano (Madrid 1999); K. Lomas et T. Collins (éd.), Bread and Circuses. Euergetism and Municipal Patronage in Roman Italy (Londres 2003); J. Andreu Pintado, Munificiencia pública en la provincia Lusitania (siglos I-IV d.C.) (Saragosse 2004). Pour l'ordre équestre en particulier : G. Wesch-Klein, 'Equites Romani und Euergetismus', in S. Demougin, H. Devijver et M.-Th. Raepsaet-Charlier (éd.), L'ordre équestre. Histoire d'une aristocratie (II siècle av. J.-C.$I I I^{e}$ siècle ap. J.-C.). Actes du colloque international organisé par Ségolène Demougin, Hubert Devijver et Marie-Thérèse Raepsaet-Charlier (Bruxelles-Leuven, 5-7 octobre 1995)(Rome 1999), 301-319. Sur l'évergétisme féminin et le rapport des femmes à l'argent: R. Van Bremen, 'Women and Wealth', in A. Cameron et A. Kuhrt (éd.), Images of Women in Antiquity (Londres-Sydney 1983), 223-242 et Ead., The Limits of Participation. Women and Civic Life in the Greek East in the Hellenistic and Roman Periods (Amsterdam 1996); M.T. Boatwright, 'Plancia Magna of Perge: Women's Roles and Status in Roman Asia Minor', in S.B. Pomeroy (éd.), Women's History and Ancient History (Chapel Hill 1991), 249-272 ; M.L. Woodhull, 'Matronly Patrons in the Early Roman Empire. The Case of Salvia Postuma', in F. McHardy 
souligner l'importance pour la vie des communautés locales puisqu'elle résulte souvent, dans leur quête d'honneurs et de prestige social, d'activités publiques détenues par ces femmes (telles que des sacerdoces ou des patronages) qui impliquent de généreuses donations, plus ou moins volontaires (summa honoraria, éventuellement accompagnée d'une ampliatio). Dans le tableau présenté en fin d'article (p. 184-186) ne figurent que les actes concrets d'évergétisme sous la forme de constructions ou de dons en argent, voire aussi en nature, au bénéfice de la communauté civique. Par ailleurs, j'ai pris en considération tous les exemples de munificentia, de merita mais aussi de liberalitates, même s'ils ne sont pas aussi explicites. Je détaille les évergésies, mais aussi leur lieu d'action, leur auteur ou leur nature. J'ai en revanche laissé ici de côté toutes les indications relatives à des dons dans un cadre privé.

Brièvement, on peut constater que seuls 42 cas (pour $75^{0}$ matrones équestres) ont été répertoriés pour les trois premiers siècles de notre ère, soit une proportion légèrement supérieure aux clarissimes ( 56 pour 1200) d'après les travaux de M.-Th. Raepsaet-Charlier qui a également recensé des exemples provenant des provinces orientales de l'Empire ${ }^{12}$. Par ailleurs, la répartition géographique en Occident, plus variée que pour les femmes sénatoriales, octroie un léger avantage aux provinces africaines $(16)^{13}$ sur l'Italie $(15)^{14}$, loin devant la seule province espagnole, la Bétique (6),

et E. Marshall (éd.), Women's Influence on Classical Civilization (Londres 2004), 75-91 ; M. Navarro Caballero, 'L'élite, les femmes et l'argent dans les provinces hispaniques', in L. de Ligt et al. (éd.), Roman Rule and Civic Life: Local and Regional Perspectives. Proceedings of the Fourth Workshop of the International Network Impact of Empire (Roman Empire c. 200 B.C.-A.D. 476), Leiden, June 25-28, 2003 (Amsterdam 2004), 389-400 ; E. Melchor Gil, 'Mujeres y evergetismo en la Hispania romana', in J.F. Rodríguez Neila (éd.), Hispania y la epigrafía romana. Cuatro perspectivas (Faenza 2009), 133-178 ; S. Destephen, 'L'évergétisme aristocratique au féminin dans l'Empire romain d'Orient', in B. Caseau (éd.), Les réseaux familiaux. Antiquité tardive et Moyen-Âge. In memoriam A. Laiou et E. Patlagean (Paris 2012), 71-91.

12 Raepsaet-Charlier 2008, op. cit. (n. 8), 1031 fournit la liste des évergésies réalisées par les clarissimes aux trois premiers siècles de notre ère : 21 en Italie, 16 pour les provinces anatoliennes ( $9+1$ [de Mytilène, comptabilisé sous «Grèce» par l'auteure] en Asie; 2 en Pisidie ; 2 en Galatie ; 1 en Lycie-Pamphylie et 1 en Cilicie), 14 [et non 13 comme elle l'écrit en p. 1031] pour les provinces africaines (11 en Proconsulaire; 3 en Numidie) puis 2 cas pour les Gaules et les Germanies (1 en Narbonnaise et 1 en Germanie supérieure) ainsi que pour l'Espagne ( 1 en Bétique et 1 en Lusitanie) et 1 pour la province d'Achaïe. S'il ne fallait prendre en compte que les témoignages issus des provinces occidentales, leur nombre s'établirait donc à 39 .

139 en Proconsulaire, 4 en Numidie, 1 en Maurétanie Tingitane et 1 en Maurétanie Césarienne.

144 exemples en région I; 2 en région III ; 1 dans les régions II, IV, V, VI, VII, VIII, IX, $\mathrm{X}$ et XI. 
suivie de la Dalmatie (3), de la Dacie (1) et de la Pannonie inférieure (1). Quant à la chronologie, elle atteste que la majorité des exemples est à dater du $\mathrm{II}^{\mathrm{e}}$ s. et ils se répartissent comme suit ${ }^{15}$ :

Table 1: Datation et localisation des manifestations évergétiques

\begin{tabular}{|c|c|c|c|}
\hline Datation: & Localisation: & Nombre: & Total: \\
\hline $\begin{array}{l}\mathrm{I}^{\mathrm{er}} \text { S. av. }-\mathrm{I}^{\mathrm{er}} \text { s. ap. } \\
\text { J.-Chr. }\end{array}$ & Italia, regio VII & 1 & 1 \\
\hline I $^{\text {er }}$ s. ap. J.-Chr. & $\begin{array}{l}\text { Hispania Ulterior Baetica } \\
\text { Italia, regio I } \\
\text { Italia, regio II } \\
\text { Italia, regio IV } \\
\text { Italia, regio IX } \\
\text { Mauretania Tingitana }\end{array}$ & $\begin{array}{l}2 \\
2 \\
1 \\
1 \\
1 \\
1\end{array}$ & 8 \\
\hline $\begin{array}{l}\text { Fin } \mathrm{I}^{\mathrm{er}}- \\
\text { début } \mathrm{II}^{\mathrm{e}} \mathrm{s} \text {. }\end{array}$ & Dalmatia & 3 & 3 \\
\hline $\mathrm{II}^{\mathrm{e}}$ s. ap. J.-Chr. & $\begin{array}{l}\text { Africa Proconsularis } \\
\text { Hispania Ulterior Baetica } \\
\text { Italia, regio I } \\
\text { Italia, regio III } \\
\text { Italia, regio V } \\
\text { Italia, regio VIII } \\
\text { Italia, regio XI } \\
\text { Mauretania Caesariensis } \\
\text { Numidia }\end{array}$ & $\begin{array}{l}5 \\
3 \\
1 \\
1 \\
1 \\
1 \\
1 \\
1 \\
1\end{array}$ & 15 \\
\hline $\mathrm{II}^{\mathrm{e}}-\mathrm{III}^{\mathrm{e}} \mathrm{s}$ & $\begin{array}{l}\text { Africa Proconsularis } \\
\text { Hispania Ulterior Baetica } \\
\text { Italia, regio III } \\
\text { Italia, regio VI } \\
\text { Numidia }\end{array}$ & $\begin{array}{l}1 \\
1 \\
1 \\
1 \\
2\end{array}$ & 6 \\
\hline $\mathrm{III}^{\mathrm{e}} \mathrm{s}$. & $\begin{array}{l}\text { Africa Proconsularis } \\
\text { Dacia } \\
\text { Italia, regio I } \\
\text { Italia, regio X } \\
\text { Numidia } \\
\text { Pannonia inferior }\end{array}$ & $\begin{array}{l}4 \\
1 \\
1 \\
1 \\
1 \\
1\end{array}$ & 9 \\
\hline
\end{tabular}

15 Raepsaet-Charlier 2008, op. cit. (n. 8), 1031: pour l'ordre sénatorial, les chiffres se répartissent comme suit : 5 cas au I ${ }^{\mathrm{er}}$ s. ; 30 au $\mathrm{II}^{\mathrm{e}} \mathrm{s}$. ; 12 à la fin du $\mathrm{II}^{\mathrm{e}}$ ou au début du $\mathrm{III}^{\mathrm{e}} \mathrm{s}$. et 9 cas au $\mathrm{III}^{\mathrm{e}} \mathrm{s}$. 
Quant aux évergésies en général, elles sont le fait de femmes seules donatrices, comme le confirment les 27 cas recensés, même s'il convient tout de même de préciser que 3 de ces exemples correspondent en réalité à des donations effectuées par plusieurs d'entre elles: mères et filles à Alba Pompeia, en Italie ${ }^{16}$, et à Seressi, en Proconsulaire ${ }^{17}$, et trois sœurs à Madaure, en Numidie ${ }^{18}$. D'autres cas (9), en revanche, confirment une pratique de l'évergétisme en couple, à l'instar de Cornelia Valentina Tucciana à Timgad, en Numidie ${ }^{19}$, d'Helvidia Priscilla à Chieti, en Italie ${ }^{20}$, ou encore de Succonia Rustica à Ostur, en Bétique ${ }^{21}$. Les exemples restants se réfèrent en général à des dons accomplis en famille.

A titre d'information, car je ne vais pas m'attarder sur ce point, on peut avancer que les motivations de ces dames n'ont pas toutes pour origine leur élection à un sacerdoce, comme dans le cas de Vettia Saturnina ${ }^{22}$ ou de Cantria Longina qui, par exemple, verse 50.000 sesterces à la res publica d'Aeclanum à l'occasion de son accession à la prêtrise ${ }^{23}$. En effet, d'autres causes, quand elles sont explicitées, peuvent être mentionnées, comme lorsqu'il s'agit de remboursements divers.

Venons-en maintenant aux dons effectués par ces matrones équestres qui, mis à part les exemples vagues et imprécis mais nets de munificentia, de merita ou de beneficia, consistent en des statues (15 exemples), souvent remboursées à l'ordo local ${ }^{24}$, mais aussi en des bâtiments imposants ${ }^{25}$, comme le résume ce tableau :

16 CIL V, 7595 (p. 771) et $A E$ 2004, 579.

17 CIL VIII, $937=11216$ (p. 2340).

18 ILAlg I, 2145.

$19 A E$ 1980, 956 ; CIL VIII, 2398 (p. 1693); CIL VIII, 2399 (p. 1693) = ILS 2753 et ILS 5579.

20 CIL IX, $3019=$ ILS 1377 .

21 CIL II, 1267 = CILA Hu, 81 .

22 CIL VIII, $4437=18596$.

23 CIL IX, 1153 = ILS 6487. R. Duncan-Jones, The Economy of the Roman Empire. Quantitative Studies (Cambridge 1974), 107-108 et 216-217 fournit des données comparatives : en Afrique, les montants vont de 90.000 à 1.000 sesterces tandis qu'en Italie, Cantria Longina fait partie du groupe de tête des donateurs qui versent 50.000 sesterces et dont les chiffres minimums sont de 6.000 sesterces. Voir aussi Briand-Ponsart 2003, op. cit. (n. 7), 84-85.

24 Dont les coûts varient, d'après les chiffres de Duncan-Jones 1974, op. cit. (n. 23), 93-99, entre 66.666 sesterces (au total 1 million de sesterces pour 16 statues) à 460 sesterces en Afrique et entre 550.000 sesterces (pour plusieurs statues) et environ 500 sesterces en Italie : $162-163$.

25 Dont les montants s'élèvent, d'après les chiffres de Duncan-Jones 1974, op. cit. (n. 23), 90-93, entre 600.00o sesterces à 500 sesterces en Afrique et entre 2 millions de sesterces et environ 672,5 sesterces en Italie : $157-162$. 
Table 2: Edifices financés par des matrones équestres

\begin{tabular}{|c|c|c|}
\hline Type de bâtiment: & Nom de la matrone équestre: & Lieu: \\
\hline $\begin{array}{l}\text { Aedicula Matris } \\
\text { Magnae }\end{array}$ & Servilia M. f. Copiesilla & Salone ${ }^{26}$ \\
\hline Balineum & $\begin{array}{l}\text { Albucia M. f. Candida et son mari } \\
\text { C. Valerius C. f. Pansa }\end{array}$ & Novare $^{27}$ \\
\hline $\begin{array}{l}\text { Balineum cum } \\
\text { omni ornatu }\end{array}$ & $\begin{array}{l}\text { Cosconia Maluginensis f. Gallitta, } \\
\text { son mari [L. Aelius Strabo] et sa } \\
\text { belle-mère Terentia A. f. }\end{array}$ & Bolsena $^{28}$ \\
\hline Basilica & $\begin{array}{l}\text { Flavia T. f. Tertulla et son mari } \\
\text { M. Flavius T. f. Quir. Fronto }\end{array}$ & Doclea $^{30}$ \\
\hline $\begin{array}{l}\text { Basilica cum } \\
\text { hypaethro }\end{array}$ & Cornelia Anulla et son fils $\left[--_{-}\right]^{31}$ & Abdera $^{32}$ \\
\hline $\begin{array}{l}\text { Macellum } \\
\text { (+ exornatio), area } \\
\text { et aménagements } \\
\text { au Capitole }\end{array}$ & $\begin{array}{l}\text { Cornelia Valentina Tucciana et son } \\
\text { mari M. Plotius Faustus }\end{array}$ & Timgad $^{33}$ \\
\hline $\begin{array}{l}\text { Porticus cum } \\
\text { statuis et uiridibus }\end{array}$ & Varia Pansina & Nola $^{34}$ \\
\hline Templum & $\begin{array}{l}\text { Antonia Valentina et son mari M. } \\
\text { Cominius Quintus }\end{array}$ & $\begin{array}{r}\text { Sarmizegetusa }^{35} \\
(\text { continued })\end{array}$ \\
\hline \multicolumn{3}{|c|}{ 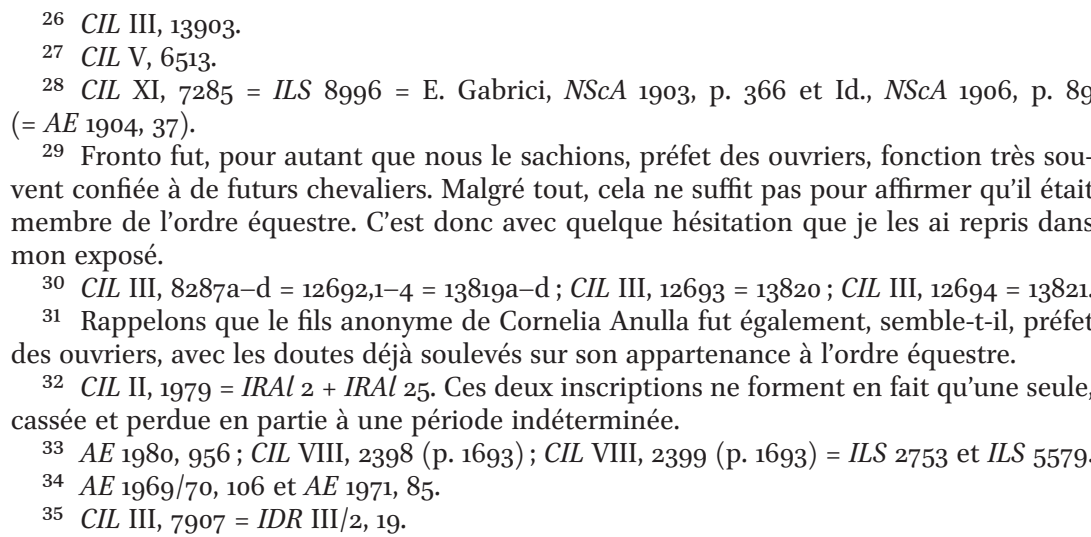 } \\
\hline
\end{tabular}


Table 2 (cont.)

\begin{tabular}{lll}
\hline Type de bâtiment: & \multicolumn{1}{c}{ Nom de la matrone équestre : } & \multicolumn{1}{c}{ Lieu : } \\
\hline$(--)$ & $\begin{array}{l}\text { Aebutia Honorata Agriana et son mari } \\
(\text { Numisius })^{36}\end{array}$ & Uchi Maius ${ }^{37}$ \\
& $\begin{array}{l}\text { Helvidia C. f. Priscilla et son mari } \\
\text { C. Vettius Marcellus }\end{array}$ & Chieti $^{38}$ \\
& $\begin{array}{l}\text { Statia Sex. f. Fida et son fils défunt } \\
\text { Q. Manlius Q. f. Rufus }\end{array}$ & Risinium $^{39}$ \\
\hline
\end{tabular}

Parfois, il peut s'agir de simples ajouts esthétiques comme des ornamenta pour l'arc de Seressi par Armenia Auge et sa fille Bebenia Pauliana ${ }^{40}$, l'exornatio du macellum de Timgad par Cornelia Valentina Tucciana et son mari ${ }^{41}$ ou la décoration avec du marbre du portique de Préneste par Pompeia Mummiana ${ }^{42}$.

Parmi toutes ces manifestations évergétiques, il en est une qui sort du lot, celle de Iunia Rustica, première flaminique perpétuelle du municipe (pré?-)flavien de Cartima, en Bétique, en raison de ses multiples actions. En effet, elle restaure les portiques, offre un espace pour la construction des bains, paie les dettes publiques, érige une statue de Mars et fait bâtir un portique à côté des bains, sur son terrain, avec une piscina et une statue de Cupidon. Enfin, elle rembourse les dépenses que l'ordo a consenties en vue de poser une statue en son honneur et en celui de son fils puis elle organise un epulum et des spectacles pour ses concitoyens ${ }^{43}$.

36 G. Wesch-Klein, Liberalitas in rem publicam. Private Aufwendungen zugunsten von Gemeinden im römischen Afrika bis $284 \mathrm{n}$. Chr. (Bonn 1990), 253-254, $\mathrm{n}^{\mathrm{0}} 11$ et 13. Et tel est également l'avis de E. Ughi, in A. Ibba et al. (éd.), Uchi Maius 2. Le iscrizioni (Sassari 2006), 213-214 où elle se montre plus nuancée que dans son article antérieur 'L'evergetismo cittadino', in M. Khanoussi et A. Mastino (éd.), Uchi Maius 1. Scavi e ricerche epigrafiche in Tunisia (Sassari 1997), 225-226 et 236, nº 16.

37 CIL VIII, $15457=26277=26278=$ Uchi Maius II, 75 .

38 CIL IX, $3019=$ ILS 1377.

39 CIL III, 1717 (p. 1028).

40 CIL VIII, $937=11216$ (p. 2340).

41 CIL VIII, 2398 (p. 1693).

42 CIL XIV, 2995. Duncan-Jones 1974, op. cit. (n. 23), 92-93 et 160-162, nous dévoile que les montants varient en général entre 800.000 sesterces et 672,5 sesterces en Italie et entre 500.000 sesterces et 500 sesterces en Afrique.

43 CIL II, $1956=I L S$ 5512. J.F. Donahue, 'Iunia Rustica of Cartima: Female Munificence in the Roman West', Latomus 63 (2004), 873-891 : coût de 60.000 sesterces selon lui. Sur la formule impensam remisit, voir S. Dardaine, 'La formule épigraphique « impensam remisit » et l'évergétisme en Bétique', Mélanges de la Casa de Velázquez 16 (1980), 39-55. 
Ce dernier exemple m'offre l'occasion d'évoquer la présence de banquets et de sportules dans le cadre de l'évergétisme qui apparaissent dans 12 cas et sont toujours complémentaires d'autres actes évergétiques dont ils matérialisent l'inauguration. Quelquefois, en de rares occasions, des montants nous sont précisés pour des distributions d'argent: Aurelia Calligenia verse ainsi 4 sesterces aux décurions à Rimini ${ }^{44}$ tandis que Varia Pansina, à Nola, remet 30 sesterces aux décurions, 20 sesterces aux Augustales, 12 sesterces aux ministri (desservants du culte de Vénus) et 4 sesterces aux personnes ressortant du populus et aux femmes ${ }^{45}$. Enfin, Aemilia Pudentilla, pour sa part, distribue 50.000 sesterces lors du mariage de son fils aîné Pontianus et de la prise de toge virile de son cadet Pudens ${ }^{46}$.

Pour en finir avec cette section consacrée à l'évergétisme, il convient d'évoquer les sommes investies dans la construction des bâtiments offerts à la communauté. Il n'est, à ce propos, pas toujours possible d'en déterminer le montant avec précision:

Table 3: Montants et justification de donations effectuées par des matrones équestres

\begin{tabular}{llll}
\hline Nom : & \multicolumn{1}{c}{ Lieu: } & \multicolumn{1}{c}{ Motif: } & Montant: \\
\hline $\begin{array}{l}\text { Albucia M. f. } \\
\text { Candida }\end{array}$ & Novare & Legs à la res publica & $200.000 \mathrm{HS}^{47}$ \\
$\begin{array}{l}\text { Septimia Polla } \\
\text { et son frère, }\end{array}$ & Leptis Magna & $\begin{array}{l}\text { Legs pour une statue } \\
\text { d'argent }\end{array}$ & $>115.000 \mathrm{HS}^{48}$ \\
$\begin{array}{l}\text { P. Septimius } \\
\text { Geta }\end{array}$ & & &
\end{tabular}

(continued)

44 CIL XI, 405 .

$45 A E 1969 / 70,106$ et $A E$ 1971, 85.

46 Apul., Apol. 87, 10-11 et Duncan-Jones 1974, op. cit. (n. 23), 105-106 et 188-198 : entre 100 et 1 sesterce par tête en Afrique - la distribution d'Aemilia Pudentilla étant la plus élevée de sa catégorie -, tandis qu'en Italie, les montants s'élèvent de 400 à 1 sesterce par tête. Sur Aemilia Pudentilla en particulier: H. Pavis d'Escurac, 'Pour une étude sociale de l'Apologie d'Apulée', Antiquités africaines 8 (1974), 89-101; A. Gutsfeld, 'Zur Wirtschaftsmentalität nichtsenatorischer provinzialer Oberschichten: Aemilia Pudentilla und ihre Verwandten', Klio 74 (1992), 250-268 et M. Lakhlif, 'La richesse et les stratégies matrimoniales en Afrique au Haut-Empire: Aemilia Pudentilla, épouse d'Apulée de Madaure', in J. González et al. (éd.), L'Africa romana. Le richezze dell'Africa. Risorse, produzioni, scambi. Atti del XVII convegno di studio. Sevilla, 14-17 dicembre 2006 (Rome 2008), 319-326.

47 CIL V, 6513.

$48 A E 1950,158=I R T 607$. Le poids de la statue est de 144 livres et 10 onces et demie (un peu moins de $47 \mathrm{~kg}$ ), volume auquel son frère et héritier ajoute un vingtième $(2,4 \mathrm{~kg}$ ) et 4 livres, 10 onces et demie $(1,617 \mathrm{~kg})$, pour un coût de plus de 115.000 sesterces selon Duncan-Jones 1974, op. cit. (n. 23), 94, le plus élevé de sa catégorie en Afrique. 
Table 3 (cont.)

\begin{tabular}{|c|c|c|c|}
\hline Nom : & Lieu : & Motif: & Montant : \\
\hline $\begin{array}{l}\text { Pompeia Q. f. } \\
\text { Trebulla }\end{array}$ & Terracine & $\begin{array}{l}\text { Legs avec pour prescription } \\
\text { une restauration du } \\
\text { monument bâti par le } \\
\text { mari, M. Iunius C. f. Gal. } \\
\text { Proculus }\end{array}$ & $100.000 \mathrm{HS}^{49}$ \\
\hline Statia Sex. f. Fida & Risinium & $\begin{array}{l}\text { Ajout au don du fils, } \\
\text { Q. Manlius Q. f. Serg. } \\
\text { Rufus }+5 \% \text { du total }\end{array}$ & $\begin{array}{l}35.000 \mathrm{HS}+ \\
13.250 \mathrm{HS}^{50}\end{array}$ \\
\hline $\begin{array}{l}\text { Armenia Auge et } \\
\text { sa fille, Bebenia } \\
\text { Pauliana }\end{array}$ & Seressi & $\begin{array}{l}\text { Ornements de l'arc } \\
\text { bâti par le fils et le } \\
\text { frère C. Memmius Felix } \\
\text { Armenianus }\end{array}$ & $25.000 \mathrm{HS}^{51}$ \\
\hline $\begin{array}{l}\text { Maria Lucina et ses } \\
\text { deux fils, L. Fulvius } \\
\text { Kastus Fulvianus et } \\
\text { [L.?] Fulvius Felix }\end{array}$ & Mustis & Ob honorem [- - - ] & $>10.000 \mathrm{HS}^{52}$ \\
\hline
\end{tabular}

A titre de comparaison, les chiffres pour les femmes de l'ordre sénatorial sont dans l'ensemble comparables, même si certains montants sont logiquement plus élevés ${ }^{53}$.

Passons maintenant aux quelques témoignages relatifs à des matrones dont les dépenses ne peuvent être mises sur le compte de l'évergétisme. Je pense à Aurelia Flavia qui nous indique explicitement qu'à Lambèse, elle a déboursé 5.000 sesterces pour faire ériger un autel en l'honneur de son défunt mari, l'officier Ulpius Longinus, ce qui rentre dans le dossier des dépenses à des fins «privées» que j'ai mentionnées au début de cet exposé54.

49 CIL X, 6309 (p. 1015). Cf. n. 42 pour les données comparatives fournies par DuncanJones 1974, op. cit. (n. 23).

50 CIL III, 1717 (p. 1028).

51 CIL VIII, $937=11216$ (p. 2340). Les chiffres donnés par Duncan-Jones 1974, op. cit. (n. 23), 92-93 et 160-162, s'élèvent à environ 800.000 sesterces et 672,5 sesterces en Italie et entre 500.000 sesterces et 500 sesterces en Afrique.

52 CIL VIII, 1578 (p. 1501). Duncan-Jones 1974, op. cit. (n. 23), 90-92 et 157-16o, nous informe que les montants varient entre 2 millions de sesterces et 6.000 sesterces en Italie et entre 600.000 sesterces et 3.000 sesterces en Afrique.

53 Raepsaet-Charlier 2008, op. cit. (n. 8), 1037.

$54 A E$ 1989, 872. Duncan-Jones 1974, op. cit. (n. 23), 99-101 et 166-171 : coûts entre plus de 80.000 sesterces et 96 sesterces en Afrique; entre 500.000 sesterces et 120 sesterces en Italie. 
D'autres montants chiffrés peuvent également être dévoilés : c'est ainsi que nous savons que la mère et la sœur de Perse avaient hérité de lui 2 millions de sesterces ${ }^{55}$. Pline le Jeune, lui, relate que son meilleur ami, Voconius Romanus, avait reçu de sa mère, en vue d'intégrer l'ordre sénatorial, un don de 4 millions de sesterces, sans que l'on sache si cette démarche louable fut couronnée de succès ${ }^{56}$. Apulée raconte que son épouse, Aemilia Pudentilla, disposait d'une fortune s'élevant à 4 millions de sesterces, qui suscita bien des convoitises ${ }^{57}$. Quant à sa dot, elle s'éleva à 300.000 sesterces, lorsqu'elle s'unit à son premier mari ${ }^{58}$. Toujours dans l'œuvre d'Apulée, nous apprenons que la grand-mère d'Herennia, épouse de Sicinius Pontianus puis de Pudens, avait reçu de son mari 3 millions de sesterces pour échapper aux dettes qu'il avait contractées ${ }^{59}$. Plus tard, nous lisons que le père d'Herennia a dû emprunter 400.000 sesterces pour son mariage avec les fils de Pudentilla ${ }^{60}$. A titre de comparaison, Apulée lui-même n'avait pas à rougir puisque son père disposait d'un patrimoine de 2 millions de sesterces, dont il hérita la moitié61.

Par ailleurs, nous connaissons un nombre important de femmes qui se déclarent héritières mais pour lesquelles il n'est fait état d'aucun montant. Tout au plus peut-on déduire que le legs a sans doute servi à financer l'érection d'une inscription destinée à commémorer leur parent décédé. Tel est le cas des matrones suivantes:

Table 4: Matrones équestres légataires de leurs proches

\begin{tabular}{lll}
\hline Nom : & \multicolumn{1}{c}{ Nom du testateur: } & \multicolumn{1}{c}{ Lieu: } \\
\hline Aelia Filonice & {$[$ M.?] Aurelius M. f. Romanus, mari } & Lambèse $^{62}$ \\
[Attia M. f. - - ] & {$[$ - Attius M. f. Te]r. Vindex, frère } & $\begin{array}{l}\text { Aix-en- } \\
\text { Provence }\end{array}$ \\
& & (continued)
\end{tabular}

55 Prob., Vita Persi 7-8. A cette somme s'est joint un montant indéterminé qui leur fut reversé par son ami L. Annaeus Cornutus.

56 Plin., Ep. 10, 4, 2-5.

57 Apul., Apol. 71, 6 et 77, 1.

58 Apul., Apol. 92, 1 et 3.

59 Apul., Apol. 75, 8.

60 Apul., Apol. 92, 2.

61 Apul., Apol. 23, 1.

62 CIL VIII, 2762.

63 CIL XII, 514 (p. 814) = 515 = ILN III, 25. Bien que l'inscription ait été mise au jour sur le territoire d'Aix-en-Provence, les Attii provenaient sans doute d'Arles. 
Table 4 (cont.)

\begin{tabular}{|c|c|c|}
\hline Nom : & Nom du testateur: & Lieu : \\
\hline $\begin{array}{l}\text { Cornelia Eulogia } \\
\text { Romanilla et ses sœurs } \\
\text { Cornelia Romanilla } \\
\text { Postumiana } \\
\text { Cornelia Valentina } \\
\text { Claudiana }\end{array}$ & $\begin{array}{l}\text { M. Cornelius Fronto Gabinianus, } \\
\text { père }\end{array}$ & Madaure $^{64}$ \\
\hline Iulia [Pie]ris & Q. Fabius Q. f. Gal. Blandus, mari & Rome $^{65}$ \\
\hline $\begin{array}{l}\text { Laecania Martina et ses } \\
\text { fils }\end{array}$ & M. Acutius PP. f. Acutianus, mari & Cluana $^{66}$ \\
\hline Longinia Saturnina & $-[-]$ Iulius [- - -], mari & Césarée 67 \\
\hline Numitoria C. f. Moschis & L. Cominius L. f. Maximus, mari & Tivoli 68 \\
\hline $\begin{array}{l}\text { Statilia Crescentilla et } \\
\text { son fils } \\
\text { Munatius Paulinus }\end{array}$ & $\begin{array}{l}\text { Munatius Paul[i]nianus, mari et père } \\
\text { des héritiers }\end{array}$ & Rome $^{69}$ \\
\hline Viatoria Taurina & C. Viatorius [-- -], père & Virunum $^{70}$ \\
\hline Vibia Maria Maxima & $\begin{array}{l}\text { P. Vibius P. f. Marianus, père } \\
\text { Reginia Maxima, mère }\end{array}$ & Rome $^{71}$ \\
\hline $\begin{array}{l}\text { Ulpia Paratiana et sa } \\
\text { fille Ulpia Valeria }\end{array}$ & $\begin{array}{l}\text { M. Val(erius) Valerianus, mari et père } \\
\text { des héritières }\end{array}$ & Brigetio $^{72}$ \\
\hline
\end{tabular}

Vient maintenant une question importante : à la vue de ces chiffres, quelle est l'origine de la fortune des parentes de chevaliers ? Conformément à la tradition aristocratique romaine, ce sont les biens fonciers qui constituent la seule source de richesse acceptable aux yeux de tous. Il ne faut donc pas s'étonner de découvrir que des parentes de chevaliers aient possédé de

64 ILAlg. I, 2145.

65 CIL VI, 3518.

${ }^{66}$ CIL IX, 5805 .

67 CIL VIII, 21037.

68 CIL XIV, 3626 = ILS $2742=$ InscrIt. IV, 1, 157. Héritière de son premier époux, elle institue à son tour son deuxième mari et le fils né de leur union comme héritiers : CIL XIV, 3628 = InscrIt. IV, 1, 158 .

${ }^{69}$ CEMC $19=A E$ 1990, 64 .

70 CIL III, $4859=$ ILLPRON 608 (cas douteux).

${ }_{71}$ CIL VI, 1636 (p. 3163, 3811 et 4723) = ILS 1361. La famille provient de Dertosa (Regio XI).

72 CIL III, 4327 = RIU III, 637 . 
telles propriétés ${ }^{73}$. Toutefois, il subsiste une difficulté, et non des moindres, l'impossibilité d'établir avec certitude que nos sources fassent clairement allusion à un domaine rural, si aucune mention n'y est faite explicitement. En outre, à la différence des familles sénatoriales, il n'est guère aisé d'identifier les lieux de résidence des chevaliers et de leurs proches, puisqu'ils n'étaient pas tenus à l'obligation de résidence à Rome. L'ordre équestre était tout aussi provincial dans son recrutement que les sénateurs, mais la présence de chevaliers dans leurs cités d'origine fut plus marquée par leur participation aux magistratures et autres sacerdoces ${ }^{74}$. Cela veut-il dire que toutes les matrones équestres attestées dans n'importe quelle cité de l'Empire y aient possédé une propriété ? Cela est fort possible mais dans le cas qui m'occupe, je ne vais prendre en considération que les exemples documentés les plus nets. Pour ce faire, le recours à l'archéologie s'avère ici la clé essentielle pour confirmer l'existence d'un domaine sur lequel a vécu ou qu'a possédé une matrone équestre ${ }^{75}$.

73 La preuve en a été fournie pour les chevaliers romains : S. Demougin, L'ordre équestre sous les Julio-claudiens (Rome 1988), 85-91; G. Paci, 'I cavalieri romani e la proprietà fondiaria dai Flavi ai Severi', in S. Demougin, H. Devijver et M.-Th. Raepsaet-Charlier (éd.), L'ordre équestre. Histoire d'une aristocratie (II siècle av. J.-C.-III e siècle ap. J.-C.). Actes du colloque international organisé par Ségolène Demougin, Hubert Devijver et Marie-Thérèse Raepsaet-Charlier (Bruxelles-Leuven, 5-7 octobre 1995) (Rome 1999), 291-300.

${ }^{74}$ La présence de sénateurs parmi les magistrats municipaux d'une cité provinciale est justement un indice pouvant certifier qu'ils y possédaient une propriété : Andermahr 1998, op. cit. (n. 7), 10. Des sénateurs titulaires de magistratures locales sont attestés durant tout le Haut-Empire en Italie et dans les provinces. Cf. W. Eck, 'La presenza delle famiglie senatorie nelle città dell'Impero romano fino al tardo III secolo', in Id. (éd.), Tra epigrafia, prosopografia e archeologia. Scritti scelti, rielaborati ed aggiornati (Rome 1996), 175-212.

${ }^{75}$ Le pionnier des travaux sur cette thématique est P.-A. Février, 'Villes et campagnes des Gaules sous l'Empire', Ktema 6 (1981), 359-372 qui s'était intéressé à la Gaule et à l'Italie du Nord en fournissant des critères pour l'identification des propriétés. Depuis lors, Andermahr 1998, op. cit. (n. 7), 7-42, a proposé une série d'indices pouvant mener à identifier les propriétés ayant appartenu à des membres de l'ordre sénatorial et qui sont, bien évidemment, valables pour les chevaliers et leurs proches, avec les nuances que j'ai soulevées. Par la suite, sur la base d'études régionales, certains savants ont présenté d'autres critères d'identification: M. Christol, 'Le patrimoine des notables en Gaule méridionale. Apports et limites de l'épigraphie', Histoire et sociétés rurales 19 (2003), 133-150 ; M. Silvestrini, 'Les biens-fonds des élites locales en Italie du sud. L'exemple des Regiones II et III (Apulie et Calabre)', Histoire et sociétés rurales 19 (2003), 51-65; E. Melchor Gil, 'Las propriedades rústicas de las élites hispano-romanas: un intento de aproximación a través de la documentación epigráfica', in J.F. Rodríguez Neila et E. Melchor Gil (éd.), Poder central y autonomía municipal: la proyección pública de las élites romanas de Occidente (Cordoue 2006), 244-252 et Id., 'Sobre las posesiones rústicas de las élites municipales de la Bética y la vinculación de sus proprietarios con determinadas comunidades cívicas', Mélanges de l'Ecole française de Rome. Antiquité 119 (2007), 435-443. 
A ce propos, en premier lieu, quelques textes littéraires sont clairs: nous savons ainsi que la mère du poète Perse, Fulvia Sisennia, possédait des biens en Ligurie, hérités de son second mari ${ }^{76}$. C'est dans les environs de Rome que nous trouvons un cas un peu plus douteux, celui de Pompeia Paulina, dont on sait qu'elle vivait dans une villa suburbaine mais qui aurait pu appartenir à son mari, Sénèque, ou à son père, le préfet de l'Annone arlésien Pompeius Paulinus ${ }^{77}$. Pour sa part, c'est dans son domaine de Vintimille, en Ligurie, que périt assassinée par les soldats d'Othon Iulia Procilla, mère d'Agricola, le beau-père de Tacite ${ }^{78}$. De son côté, Plinia, mère de Pline le Jeune, était possessionnée dans les environs du lac de Côme avec des propriétés que son fils a héritées ${ }^{79}$. Enfin, en ce qui concerne la mère de Voconius Romanus, nous savons qu'elle avait renoncé à la possession de biens fonciers, sans doute dans la région de Sagonte, qu'elle a remis à son fils pour qu'il puisse disposer du patrimoine nécessaire en vue d'accéder au Sénat ${ }^{80}$.

L'épigraphie, pour sa part, nous renseigne non seulement sur l'existence de domaines ruraux mais aussi très souvent sur leur emplacement, confirmé par plusieurs indices convergents. Quelquefois, la mention est explicite, comme pour Roia Titinia, son mari et ses fils à Uchi Maius, où une architrave indique l'entrée des praedia leur appartenant ${ }^{81}$. Le fundus Flauianus aurait pu, quant à lui, appartenir à Aelia Flaviana, originaire de Cordoue $^{82}$ tout comme le fundus Nasennianus appartiendrait à la famille de Nasennia Iusta de Bénévent ${ }^{83}$. Mais là rien n'est moins sûr.

76 Schol. Pers. 6, 6.

77 Tac., Ann. 15, 6o, 7 .

78 Tac., Agr. 7, 2.

79 Plin., Ep. 7, 11, 5 .

80 Plin., Ep. 10, 4, 3 .

81 A. Merlin et L. Poinssot, Les inscriptions d'Uchi Maius d'après les recherches du capitaine Gonduin (Paris 1908), 108-112, nº 173 = ILS 6024 et Add., p. CLXXXVI. L'inscription figure aussi sous le numéro 26415 du CIL VIII, mais les éditeurs y omettent la titulature équestre de (Pullaienus) Celsinius Pupianus, mari de Roia Titinia.

82 M. Gallego Franco, 'Participación de la mujer hispanorromana en la producción y comercio del aceite bético', in AA.VV., Congreso internacional "Ex Baetica amphorae". Conservas, aceite y vino en el Imperio romano (Sevilla - Écija, 17 al 20 de diciembre de 1998), IV (Écija 2000), 1272, n. 37 suggère cette hypothèse. Aelia Flaviana est connue par CIL II, $2224=I L S 6905=C I L I^{2} / 7,282$.

83 M.R. Torelli, Beneventum (Rome 2002), 327, n. 99 et 335, n. 129. Signalons qu'à l'instar de Cornelia Anulla, Nasennia Iusta est apparentée à un praefectus fabrum, dont l'appartenance à l'ordre équestre est plus que douteuse : CIL IX, 1646 = ILS 6498. 
Ailleurs, ce sont des indices et des données archéologiques qui, combinés aux inscriptions et à la toponymie, nous certifient l'existence de propriétés rurales :

Table 5: Matrones équestres propriétaires de domaines fonciers

Nom : Lieu :

Acilia Plecusa

Albucia M. f. Candida

Atilia L. f. Vera et son mari L. Aemilius L. f. Paternus

Caecilia L. f. Macrina, avec son mari P. Senecius P. f. Fab. Garrulus et son fils homonyme

Carbetania Maxima et son père

P. Carbetanius Rufus

Domitia Sex. f., et ses enfants L. Domitius L. f. Vol. Magu[- - - ], L. Domitius L. f. Vol. Celer et [-] Domitius L. f. Vol. Macer

Iulia [- - - ]a et son mari, dont on ne peut reconstituer le nom
Singilia Barba ${ }^{84}$

Albuzzano (près de Pavie) ${ }^{85}$

Perolet (près d'Aeso) ${ }^{86}$

Rodengo (près de Brescia) ${ }^{87}$

Ferentinum $^{88}$

Rognes (près d'Aix-en-

Provence $)^{89}$

Cuers (près d'Arles) ${ }^{90}$

(continued)

84 CIL II $/ 5,830$ : Melchor Gil 2006, op. cit. (n. 75), 254 et Melchor Gil 2007, op. cit. (n. 75), 436 et 440. Acilia Plecusa fut l'épouse d'un praefectus fabrum.

85 Albuzzano, situé à une dizaine de $\mathrm{km}$ au nord-est de Pavie, peut indiquer la présence d'une propriété des Albucii, gens à laquelle était rattachée Albucia Candida qui y avait détenu le flaminicat de la divine Sabine (cf. CIL V, 6514). E. Gabba, "Ticinum: dalle origini alla fine del III sec. d. C.', in AA.VV., Storia di Pavia, I, L'età antica (Pavie 1984), 233 et M.S. Bassignano, 'Sacerdozi femminili nell'Italia settentrionale', Atti e memorie dell'Ateneo di Treviso 12 (1994-1995), 74, $\mathrm{n}^{\circ} 11$.

${ }^{86} C I L$ II, 4461 (p. 981) = ILS $2661=I R C$ II, 54. Atilia L. f. Vera était sans doute la fille ou la petite-fille du primipile Atilius Verus et l'épouse de L. Aemilius L. f. Gal. Paternus, préfet des ouvriers, centurion dans diverses légions puis primipile. Son rang équestre n'est donc pas avéré.

87 Cf. InscrIt. X/5, 1005. Pour cette hypothèse, S. Mollo, La mobilità sociale a Brescia romana (Milan 200o), 56 et 94 et F. Tassaux, 'Élites locales, élites centrales. Approche économique et sociale des grands propriétaires au nord de l'Italie romaine (Brescia et Istrie)', Histoire et sociétés rurales 19 (2003), 117.

${ }^{88}$ CIL X, $8387=$ CIL VI, 1602 (p. 3163, 4718).

89 ILN III, 243-245.

90 CIL XII, 392 et $A E$ 1999, 1016. 
Table 5 (cont.)

Nom :

Lieu :

Kharisia C. f. Magn[- - - ], son mari

C. Caecilius Victor et ses fils C. Caecilius

Victor et T. Flavius Felix

Nonia M. f. Arria Hermionilla et son mari Sex. Valerius Sex. f. Fab. Poblicola Vettillianus

Numisia Victorina et son mari [-] Furius P. f. Cambrils (près de Tarragone) ${ }^{93}$ Quir. [M]ontanus

Pompeia T. f. Sextina et son père

T. Pompeius T. f. Trom. Albinus

Publicia Pusinna et son mari M. Nonius

Cornelianus

Vettia C. f. Tranquilla, son mari Q. Sextius C. f. Pap. Martialis et ses fils T. Sextius C. f. Pap. Alexander et C. Sextius Martialis

[- - - ]ia Iusta et son mari [-] Salvius T. f. Pap. Rufinus Minicius Opimianus
Anguillara Sabazia (près de

Rome $)^{91}$

Urago Mella (près de Brescia) ${ }^{92}$
Arco (près de Brescia) ${ }^{95}$

Collatia (près de Rome) ${ }^{96}$

Grottaferrata (près de Tusculum $)^{97}$

D'autres types d'inscriptions, votives celles-là, peuvent également servir de preuve pour établir l'existence de propriétés. Dans le cas de Desideratia Desiderata et de son mari C. Iustinius Favor, nous savons que leur dédicace à Jupiter Très bon, Très grand et à Junon Regina fut érigée in $s u o^{98}$. Il en va de même pour la dédicace à Hygie posée à l'instigation de

91 CIL XI, 448* et $A E$ 1977, 259.

92 Cf. CIL V, 4484 = InscrIt. X/5, 275: Tassaux 2003, op. cit. (n. 87), 118.

$93 A E 1987,731=$ IRAT 27.

94 CIL XII, $2327=I L S 6995=I L N$ V/2, 515. Le toponyme Arbin, sis près de Montmélian, semble indiquer la présence d'une propriété ayant appartenu à cette famille. Cf. en dernier lieu, B. Rémy et F. Kayser, Les Viennois hors de Vienne. Attestations (épigraphiques, littéraires et papyrologiques) de l'activité des Viennois(es) hors de leur cité (Bordeaux 2005), 50.

95 Cf. InscrIt. X, 5, 1070 et CIL V, 4993 = InscrIt. X, 5, 1071 : Tassaux 2003, op. cit. (n. 87), 119.

96 CIL VI, 28715; $A E$ 1974, 143a = CIL VI, 41289 et $A E$ 1974, 143b. La famille semble provenir de Mactar, en Afrique Proconsulaire (cf. G. Alföldy, in CIL VI, ad 41289, p. 5038).

$97 A E$ 1906, 80. Le couple devait posséder un bien à proximité du lieu où fut découvert le fragment d'inscription les mentionnant; le souvenir de l'existence de leur propriété semble s'être maintenu dans l'onomastique à travers les siècles : Andermahr 1998, op. cit. (n. 7), 343-344, nº 346 et M. Valenti, Ager Tusculanus (Florence 2003), 83-84 et 289 (Forma Italiae 41).

98 CIL XIII, 7269. 
Cornelia Valentina Tucciana et son mari à leur domicile privé99. A l'inverse, un hommage dressé en l'honneur de Iulia Festilla à Yverdon par les uikani atteste peut-être un domaine ${ }^{100}$. On peut en dire de même pour Arminia Paulina à Timgad ${ }^{101}$ ainsi que pour Tertia Romana et son mari à Maudach (près du castellum de Rufiniana) ${ }^{102}$.

Le contexte archéologique peut également fournir des éléments de confirmation, comme dans le cas de Valeria Rufina et de son fils pour lesquels l'existence d'une villa a été conjecturée à proximité du lieu où le sarcophage de ce dernier a été mis au jour ${ }^{103}$. L'archéologie, combinée à la présence d'épitaphes d'esclaves, révèle l'emplacement de propriétés d'Arrecina Tertulla en Apulie ${ }^{104}$. En revanche, on ne saurait en dire de même pour Claudia Gallitta, originaire de Rusicade, en Numidie, mais qu'une épitaphe en l'honneur d'un oỉxovópos dénommé Philon, provenant des environs de Nicée, en Bithynie, mentionnerait ${ }^{105}$. Rien n'est moins sûr car l'homonymie est possible et d'autres arguments qu'il n'est pas lieu ici de rappeler rendent délicate cette interprétation ${ }^{106}$.

Les fistules de plomb, elles aussi, attestent, pour leur part, des résidences urbaines suffisamment étendues et appartenant à des personnalités fortunées qui ont reçu le droit de posséder une connexion avec le réseau d'approvisionnement en eau. Cela serait le cas, selon W. Eck, d'Aelia Marciana, participante aux Jeux séculaires de l'an 204 mais un problème d'identification soulevé par C. Bruun rend difficile l'interprétation du chercheur allemand ${ }^{107}$.

\footnotetext{
99 R. Cagnat, Bulletin archéologique du comité des travaux historiques (1901), CCX.

100 CIL XIII, $5064=$ ILS 7010 .
}

101 A. Ballu, 'Rapport sur les travaux de fouilles exécutés en 1906 par le service des monuments historiques en Algérie', Bulletin archéologique du comité des travaux historiques (1907), 277 : Briand-Ponsart 2003, op. cit. (n. 7), 82, n. 15.

102 CIL XIII, 11695: W. Spickermann, "Mulieres ex voto": Untersuchungen zur Götterverehrung von Frauen im römischen Gallien, Germanien und Rätien (1.-3. Jahrhundert n. Chr.) (Bochum 1994), 252.

103 AE 1965, 259 : Y. Burnand, Primores Galliarum, I, Méthodologie (Bruxelles 2005), 105 et 300 .

${ }^{104}$ CIL IX, 6183 = M. Chelotti, 'Epigrafi latine monumentali di Ruvo', in M. Pani (éd.), Epigrafia e territorio. Politica e società. Temi di antichità romane, II (Bari 1987), 73-74, $\mathrm{n}^{\circ} 14$ : Andermahr 1998, op. cit. (n. 7), 159-160.

$105 I K-9 / \mathrm{I}, 196$.

106 L'hypothèse de l'identification de la matrone équestre de Numidie avec son homonyme de Bithynie est soutenue par W. Eck, 'Miscellanea prosopographica', Zeitschrift für Papyrologie und Epigraphik 42 (1981), 254-256.

107 CIL XV, 7407. Cf. W. Eck, 'Die Fistulae aquariae der Stadt Rom. Zum Einfluss des sozialen Status auf administatives Handeln', in AA.VV., Atti del colloquio internazionale AIEGL su «Epigrafia e ordine senatorio ». Roma, 14-20 maggio 1981, I (Rome 1982), 210, n. 64 = Id., Die Verwaltung des römischen Reiches in der hohen Kaiserzeit. Ausgewählte und 
Enfin, un terrain appartenant à Viria Rustica, situé à Thugga et sur lequel fut bâti un sanctuaire en l'honneur de Tibère, dénote qu'elle possédait des terres dans ce pagus dépendant de la cité de Carthage ${ }^{108}$.

Avec toutes ces attestations de biens fonciers, il convient maintenant de se demander quelles fonctions premières occupaient ces propriétés rurales? Manifestement, il s'agissait de résidences de villégiature mais aussi de villas d'exploitation. A cet égard, l'exemple d'Aemilia Pudentilla est sans doute le plus clair puisque nous lisons, sous la plume d'Apulée, qu'elle résidait de temps à autre dans une villa suburbaine, dans laquelle d'ailleurs elle se maria avec l'écrivain ${ }^{109}$. Elle possédait en outre une domus à Oea avec vue sur mer ainsi que d'autres biens distants de 100 milles de la cité côtière ${ }^{110}$. En outre, Aemilia Pudentilla qui sait gérer ses biens avec compétence, comme le reconnaît Apulée lui-même ${ }^{111}$, a acquis, avec l'accord de son tuteur, Cassius Longinus, un petit champ à 60.000 sesterces $^{112}$.

A la vue de tous ces témoignages, on peut constater que des femmes disposaient de plein droit des biens fonciers qu'elles acquéraient ou transmettaient par le biais d'héritage, par mariage ou par achat direct. Rien, juridiquement, ne s'y opposait: sans entrer dans les détails, une femme, sui iuris ou bien mariée cum manu ou sine manu, conservant dans ce cas un lien d'agnation avec sa famille paternelle, héritait de son père au même titre que ses frères. Son patrimoine dans le cas d'une union sine manu, était dissocié de celui du conjoint qui ne possédait aucun droit dessus. En revanche, pour transmettre un legs, une femme devait toujours rédiger un testament.

Puisqu'il est clair que les femmes disposaient des biens fonciers en leur nom propre, il convient maintenant de se demander quels types d'activité y ont-elles développés.

Fort logiquement, l'agriculture ainsi que l'exploitation du bétail constituent les premières productions dans ces propriétés. Une nouvelle fois, Apulée le signale pour son épouse, Aemilia Pudentilla, quand elle léga à ses fils une partie de l'héritage de leur père auquel ils avaient droit : celui-ci

erweiterte Beiträge, II (Bâle-Berlin 1997), 259, n. 74 et C. Bruun, The Water Supply of Ancient Rome. A Study of Roman Imperial Administration (Helsinki 1991), 94, n. 75.

108 CIL VIII, 26518 = ILTun. 1402 = ILAfr. 519 = DFH 25 .

109 Apul., Apol. 87, 10.

110 Apul., Apol. 72, 6 et 44, 6.

111 Apul., Apol. 87, 7 .

112 Apul., Apol. 101, 5 . 
consistait en des terres de qualité variable, une vaste maison, une grande quantité de blé, d'orge, de vin, d'huile d'olive et d'autres produits du sol, au moins 400 esclaves et des troupeaux de valeur non négligeable ${ }^{113}$. En Egypte, vers le milieu du $\mathrm{III}^{\mathrm{e}}$ s., Aurelia Demetria, matrona stolata mariée au chevalier Aurelius Appianus a, quant à elle, loué un terrain dans l'Arsinoïte ${ }^{114}$. Par ailleurs, d'autres papyrus appartenant aux archives de Heroninos, détaillent une liste de comptes la concernant et relatifs à ses exploitations ${ }^{115}$. Parmi les autres sources de revenus, la participation dans la production et la commercialisation de l'huile d'olive ne peut être sousestimée $^{116}$. De fait, cette hypothèse a été maintes fois évoquée pour Iunia D. f. Rustica, la riche évergète de Cartima ${ }^{117}$, car elle semble être une proche parente du mercator olearius D. Iunius R[- - ], connu par des marques d'amphores datées entre $120-125$ de notre ère ${ }^{118}$. Plus récemment, a été mis au jour à Séville un autel funéraire de marbre blanc du début du $\mathrm{II}^{\mathrm{e}} \mathrm{s}$., relatif à un D. Iunius Rusticus, contemporain du précédent, à moins qu'il ne s'agisse du même personnage ${ }^{119}$. Dans ce cas-là, ces attestations confirmeraient la participation de cette famille dans la production et la commercialisation de l'huile d'olive à partir de ports et centres économiquement liés ${ }^{120}$. En effet, tant la situation de Cartima sur l'axe routier Cordoue-Málaga que la possible identification de son mari, C. Fabius Fabianus, avec un homonyme d'Acinipo ${ }^{121}$, rendent même plausibles la

113 Apul., Apol. 93, 3-4.

114 P.Flor. I, 16 (23 février 239).

115 P.Flor. I, $100\left(23^{-232}\right)$; P.Laur. I, $11(248-258)$ et plus récemment P.Prag. III, 236 (octobre 259). Sur Heroninos: D. Rathbone, Economic Rationalism and Rural Society in Third-century A.D. Egypt. The Heroninos Archive and the Appianus Estate (Cambridge 1991).

116 Sur ce thème, S. Morretta, 'Donne imprenditrici nella produzione e nel commercio dell'olio betico (I-III sec. d. C.)', Saitabi 49 (1999), 229-245 et Gallego Franco 2000, op. cit. (n. 82), 1269-1279. Pour une liste, G. Chic, Datos para un estudio socioeconómico de la Bética (Écija 2001), 471-493.

117 CIL II, $1956=$ ILS 5512 .

118 CIL XV, 3914. Cf. G. Chic, 'Los centros productores de las ánforas con marcas de L.F.C.', Hispania antiqua 18 (1994), 218-219.

119 S. Ordóñez Agulla et S. García-Dils de la Vega, 'Nuevas inscripciones de Romulenses. Con un apéndice sobre el paisaje periférico septentrional de Romula Hispalis', Romula 3 (2004), 154-162. Ces liens seraient renforcés par la présence à Séville de Fabii Fabiani, porteurs de la même onomastique que le mari de Iunia Rustica.

120 Ordóñez Agulla et García-Dils de la Vega 2004, op. cit. (n. 119), 161-162.

121 CIL II, 1356. 
participation de Iunia Rustica et de ses proches dans des activités commerciales en liaison avec l'annone ${ }^{122}$.

Pour ce qui est plus concrètement des intérêts non agricoles, d'autres sources, d'un type un peu particulier et exclusivement italien dans leur répartition, nous permettent de prendre connaissance de la présence de matrones équestres en tant que dominae documentées par le biais d'estampilles sur briques provenant de biens en général acquis ou transmis au moyen d'héritages à des parents relativement proches ${ }^{123}$. L'étude de ces marques nous confronte à un matériau qui nous est parvenu dans un état pratiquement intact, sans avoir souffert les aléas de la transmission qu'ont généralement subis les autres types d'inscriptions depuis l'Antiquité. Par conséquent, les marques de briques, où apparaît d'ailleurs un nombre relativement important de femmes, permettent d'élaborer des réflexions statistiques ${ }^{124}$. Ces marques, découvertes sur sites archéologiques, posent divers problèmes d'identification en ce qui concerne le statut des personnages qui figurent sur les sceaux : il n'est parfois en effet guère aisé de distinguer entre le dominus/a investisseur ainsi que propriétaire des figlinae ou du domaine (praedia) où a été extraite l'argile et l'officinator, dont les rôles sont variés mais qui est chargé de la production des briques ${ }^{125}$.

122 G. Chic, 'Nuevos datos económicos sobre el senador hispalense Fabius Iulianus', in C. Alonso del Real et al. (éd.), Urbs aeterna. Actas y colaboraciones del coloquio internacional, Roma entre la literatura y la historia. Homenaje a la profesora Carmen Castillo (Pampelune 2003), 394-395 et E. García Vargas et J. Martínez Maganto, 'Fuentes de riqueza y promoción social de los negotiantes salsarii béticos en el alto imperio romano. Una aproximación diacrónica', Archivo español de arqueología 82 (2009), 146.

123 P. Setälä, Private Domini in Roman Brick Stamps of the Empire. An Historical and Prosopographical Study of Landowners in the District of Rome (Helsinki 1977), 230-241. Dans une étude récente, P. Setälä nuance fortement ses assertions relatives aux héritages qu'elle considérait comme le seul moyen d'acquérir des biens fonciers. Elle estime désormais que la part des legs, sans être totalement négligeable, n'est plus aussi prépondérante comme elle le pensait initialement. De même, elle revoit aussi la place dévolue aux domini/ ae dont le rôle fut plus actif dans la production des briques, en ne se contentant pas d'être de simples propriétaires fonciers vivant de leurs rentes : Setälä 2002, op. cit. (n. 7). Pour une présentation succincte de cette thématique, C. Bruun, 'La ricerca sui bolli laterizi presentazione generale delle varie problematiche', in C. Bruun et F. Chausson (éd.), Interpretare i bolli laterizi di Roma e della valle del Tevere. Produzione, storia economica e topografia. Atti del convegno all'Ecole française de Rome e all'Institutum Romanum Finlandiae, 31 marzo e 1 aprile 2000 (Rome 2005), 3-24.

124 Setälä 1977, op. cit. (n. 123), 11. Voir déjà T. Helen, Organization of Roman Brick Production in the First and Second Centuries A.D. An Interpretation of Roman Brick Stamps (Helsinki 1975), 13.

125 Helen 1975, op. cit. (n. 124), 89-130 ; Setälä 1977, op. cit. (n. 123), 13-16 et Setälä 2002, op. cit. (n. 7), 184 ; J.J. Aubert, Business Managers in Ancient Rome. A Social and Economic Study of Instores, 250 B.C.-A.D. 250 (Leyde-New York-Cologne 1994), 224-236 et Id., 'L'estampillage des briques et des tuiles : une explication juridique fondée sur une appro- 
Qui plus est, une autre incertitude, et non des moindres, se fait jour, dans la mesure où il est difficile de certifier que les dames, fort présentes dans cette documentation au point d'être recensées sur un tiers des attestations ${ }^{126}$, étaient apparentées à des chevaliers, eux-mêmes peu nombreux et difficilement identifiables en tant que tels ${ }^{127}$. Dans sa prosopographie, confrontée à ce même dilemme, M.-Th. Raepsaet-Charlier avait pris le parti d'en faire presque toujours des clarissimes, en s'appuyant sur les données statistiques ${ }^{128}$ mais aussi sur l'onomastique, notamment, quand elle paraît conforter cette extraction ${ }^{129}$. Dans certains cas, le doute subsiste $^{130}$. En revanche, quelques femmes sont clairement apparentées à des chevaliers : il s'agit de Claudia Capitolina ${ }^{131}$, fille du préfet d'Egypte Claudius Balbillus ${ }^{132}$ et épouse d'un autre préfet d'Egypte, M. Iunius Rufus attesté à l'époque de Trajan ${ }^{133}$, et de Cusinia Gratilla ${ }^{134}$, documentée entre les années 120 et 146-147, proche parente (fille ?) de L. Cusinius Messalinus, officier et procurateur originaire d'Ephèse ${ }^{135}$.

Dans le cas qui nous occupe, une autre difficulté à laquelle nous sommes confronté est d'établir la place de ces dames «entrepreneurs», pour reprendre l'expression de P. Setälä ${ }^{136}$, dans la production de briques. Etant donné que les femmes disposaient en général d'une indépendance économique (mâtinée toutefois par la présence d'un tuteur) ${ }^{137}$, elles étaient libres de gérer leurs biens et d'en acquérir d'autres. Les biens fonciers étaient fort recherchés et pouvaient, de ce fait, faire l'objet de transactions. De judicieux investissements, pour poursuivre une «tradition»

che globale', in C. Bruun et F. Chausson (éd.), Interpretare i bolli laterizi di Roma e della valle del Tevere. Produzione, storia economica e topografia. Atti del convegno all'Ecole française de Rome e all'Institutum Romanum Finlandiae, 31 marzo e 1 aprile 2000 (Rome 2005), 53-59. Certains de ces officinatores sont des femmes: Helen 1975, op. cit. (n. 124), 112-113 (qui sous-estime leur rôle); Aubert 1994, op. cit. (n. 125), 224-226; Setälä 2002, op. cit. (n. 7), 198-200.

126 Setälä 1977, op. cit. (n. 123), 11 et 211 ; Setälä 2002, op. cit. (n. 7), 184. Voir aussi Helen 1975, op. cit. (n. 124), 23.

127 Setälä 1977, op. cit. (n. 123), 223-224.

128 Setälä 1977, op. cit. (n. 123), 211 et 250-256.

129 Cf. M.-Th. Raepsaet-Charlier, Prosopographie desfemmes de l'ordre sénatorial (I ${ }^{\text {er }}-I^{e}$ siècle) (Louvain 1987), 16 et n. 6.

130 Cf. p. ex. Aelia Severa : Setälä 1977, op. cit. (n. 123), 49-5o.

131 CIL XV, 7520 et $P I R^{2} \mathrm{C} 1086$.

$132 P^{2} \mathrm{C}_{13} ; C_{15} ;$ PME $\mathrm{C}_{124}$ et $C J C_{538 .}$

133 Setälä, 1977 op. cit. (n. 123), $94-95$ et $P I R^{2}$ I 812.

$134 P^{2} \mathrm{C} 1631$.

135 Setälä 1977, op. cit. (n. 123), 105 et $P^{2} R^{2}$ C 1627 ; CP 80 ; PME C 262.

136 Setälä 2002, op. cit. (n. 7), 181.

137 Gourévitch et Raepsaet-Charlier 2001, op. cit. (n. 7), 66-68. 
familiale acquise par héritage ou bien par volonté propre de celles-ci ${ }^{138}$, contribuaient dans tous les cas à y produire de juteux bénéfices. Pour ce qui est de la production de briques, un contrat devait être passé entre le dominus/a, propriétaire des figlinae (unités de productions) et l'officinator chargé de fabriquer les briques qui restent propriété du dominus/a qui contrôlait cette production ${ }^{139}$. Comme l'affirme P. Setälä, les femmes jouaient un rôle actif, parfois par le biais d'une societas avec leur mari ou leur frère ${ }^{140}$, en ne se contentant pas de poursuivre l'entreprise commencée par leurs pères ou maris mais en investissant elles-mêmes, de leur plein gré, en vue de produire en masse des briques, comme certaines le firent en 123, pour répondre à la demande ${ }^{141}$.

Enfin, dans un registre différent, il convient de signaler que d'autres sources de revenus pouvaient provenir de la location d'immeubles comme ce que firent Cornelia Valentina Tucciana et son mari à Timgad : à côté du marché qu'ils y firent bâtir, le couple fit édifier sur un terrain de $75 \mathrm{~m} \times$ $36 \mathrm{~m}$ la plus luxueuse maison de la colonie à laquelle furent adossées des boutiques dont la location augmentait les rentrées ${ }^{142}$.

En conclusion, on peut affirmer que les femmes apparentées aux chevaliers, en dépit des difficultés d'identification, furent loin de jouer un rôle passif dans la vie économique de leurs cités ${ }^{143}$. Généralement à la tête de leur patrimoine, basé sur des biens fonciers, elles savent l'administrer avec discernement et sagesse pour l'accroître par des acquisitions et en faire bénéficier leurs proches, au moyen d'héritages ou grâce à leur dot ${ }^{144}$. Leurs concitoyens également en jouissent par le biais de l'évergétisme,

138 Setälä 2002, op. cit. (n. 7), 192-195.

139 Setälä 2002, op. cit. (n. 7), 183-184.

140 Setälä 2002, op. cit. (n. 7), 192.

141 Setälä 2002, op. cit. (n. 7), 186-189 qui nuance ainsi les dires d'Aubert 1994, op. cit. (n. 125), 419, qui tend à minimiser la capacité des femmes à pouvoir se livrer à de telles activités.

142 Briand-Ponsart 2003, op. cit. (n. 7), 87.

143 Cette recherche pose aussi en substance la question de savoir quelle était la part du patrimoine de ces dames qui était consacrée à l'évergétisme. L'exemple d'Aemilia Pudentilla, exceptionnel à tous points de vue, permet de se faire une idée puisque nous savons qu'elle disposait d'une fortune estimée à 4 millions de sesterces au moins (Apul., Apol. 71,6 et 77,1$)$ et qu'elle a distribué 50.000 sesterces lors de la prise de toge virile de Pudens et du mariage de Pontianus (Apul., Apol. 87, 10-11). Autrement dit, Pudentilla a distribué à cette occasion-là $1,25 \%$ de son patrimoine.

144 Cf. Sen., Dial. 12, 14, 3. Cf. aussi Navarro Caballero 2004, op. cit. (n. 11), 392 et 397. En outre, depuis le règne de l'empereur Antonin, il fut permis aux épouses d'accorder un don à leurs maris afin de les aider à accéder au Sénat et à l'ordre équestre ou bien à organiser des jeux: D. 24, 1, 40-42 (extraits des écrits d'Ulpien, de Licinius Rufinus et de Gaius) et Ulp., Reg. 7, 1. 
comme il convient à des personnalités respectables et elles en retirent honneurs et prestige qui rejaillissent sur leurs proches parents ${ }^{145}$. Tout cela est conforme aux pratiques économiques propres aux femmes de l'élite qui tirent profit des revenus de leurs biens sans devoir y travailler de leurs mains ${ }^{146}$. Cela n'est toutefois pas le cas de toutes les matrones équestres, telles que l'aïeule du préfet du prétoire Nymphidius Sabinus, couturière à gages ${ }^{147}$, ou encore Nymphidia, la mère de ce même Sabinus ${ }^{148}$, accusée à tort ou à raison d'être une prostituée, à la différence de Vistilia que le fit publiquement savoir ${ }^{149}$ : elles «gagnent» leur vie et sont « rémunérées » pour ce faire, contrairement à ce qui sied à des dames de la haute société, mais cela confirme pleinement l'hétérogénéité de l'extraction et du statut social des personnes apparentées à des chevaliers, si souvent soulignée par S. Demougin pour les membres de l'ordre équestre. Par ailleurs, les considérants du SC de Larinum (sur le fait de se louer pour monter sur scène ou combattre dans l'arène) semblent refléter une réalité que la Loi ne pouvait accepter, au nom de la respectabilité des ordines supérieurs ${ }^{150}$. Les matrones équestres, par conséquent, s'adonnaient à des pratiques économiques variées, certaines plus répréhensibles que d'autres mais, en fin de compte, très souvent sur la place publique, loin du foyer où elles se devaient d'être confinées.

145 Cf. Melchor Gil 2009, op. cit. (n. 11).

146 Rien de surprenant puisque sénateurs et chevaliers, et a fortiori leurs parentes, bien que généralement plus discrètes dans nos sources, possédaient des intérêts économiques communs et leurs activités publiques étaient identiques. Voir Andreau 1999, op. cit. (n. 9) et Raepsaet-Charlier 2005, op. cit. (n. 2).

147 Plu., Galb. 9, 2.

148 Plu., Galb. 9, 1-4 et Tac., Ann. 15, 72, 1-2.

149 Tac., Ann. 2, 85, 1-4.

150 Sur le sénatus-consulte de Larinum : C. Ricci, Gladiatori e attori nella Roma giulioclaudia. Studi sul senatoconsulto di Larino (s. l. 2006). Rappelons que les sources rapportent à maintes reprises que des dames apparentées à des sénateurs et à des chevaliers montaient sur scène et combattaient dans l'arène lors de manifestations organisées à l'instigation ou en présence de l'empereur. On peut à bon droit se demander si elles étaient rémunérées pour cela : Tac., Ann. 15, 32 ; Suet., Nero 4, 2 ; Dio Cass. 55, 10, 11 ; 60, 7, 1; 61, $17,3$. 


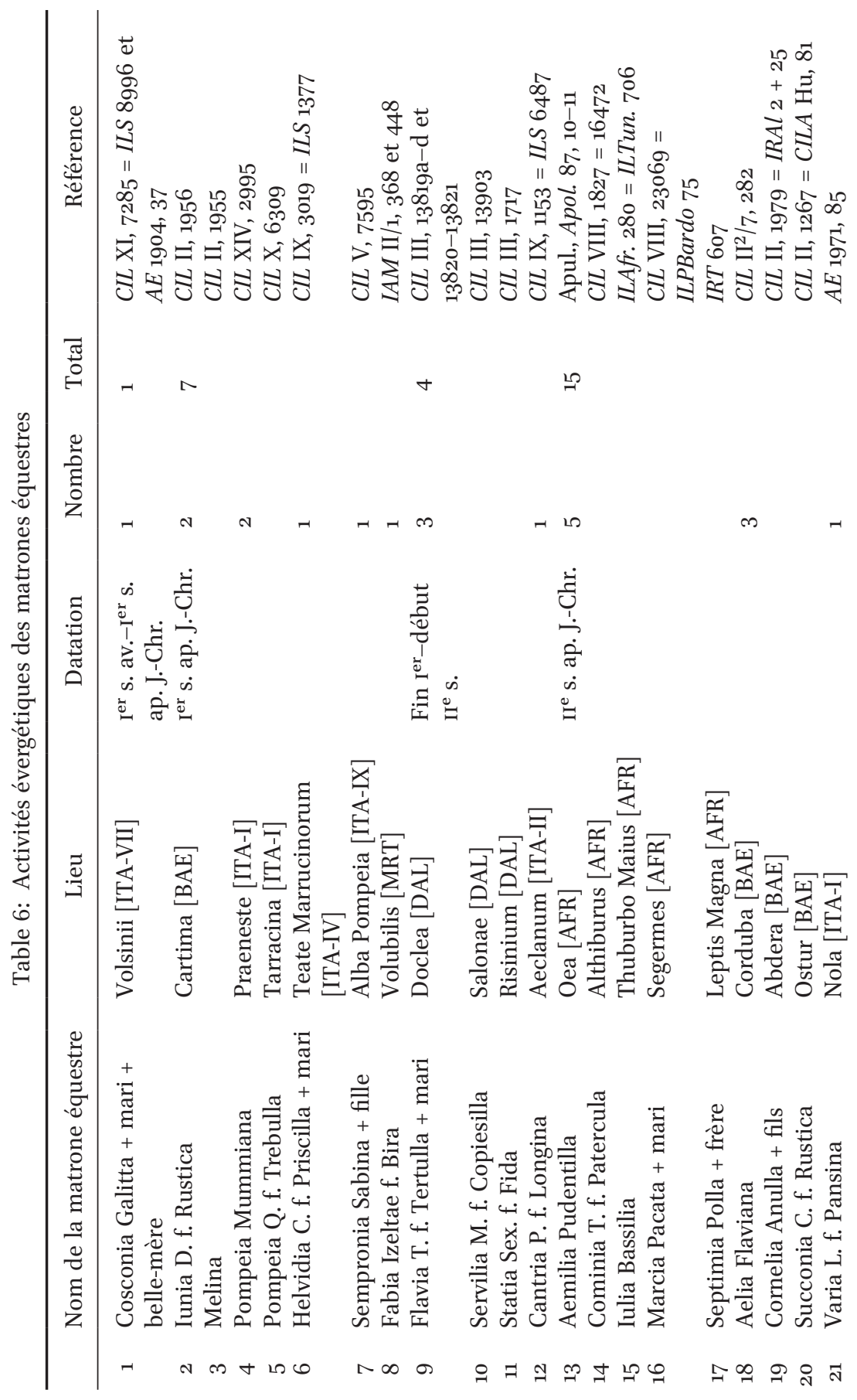




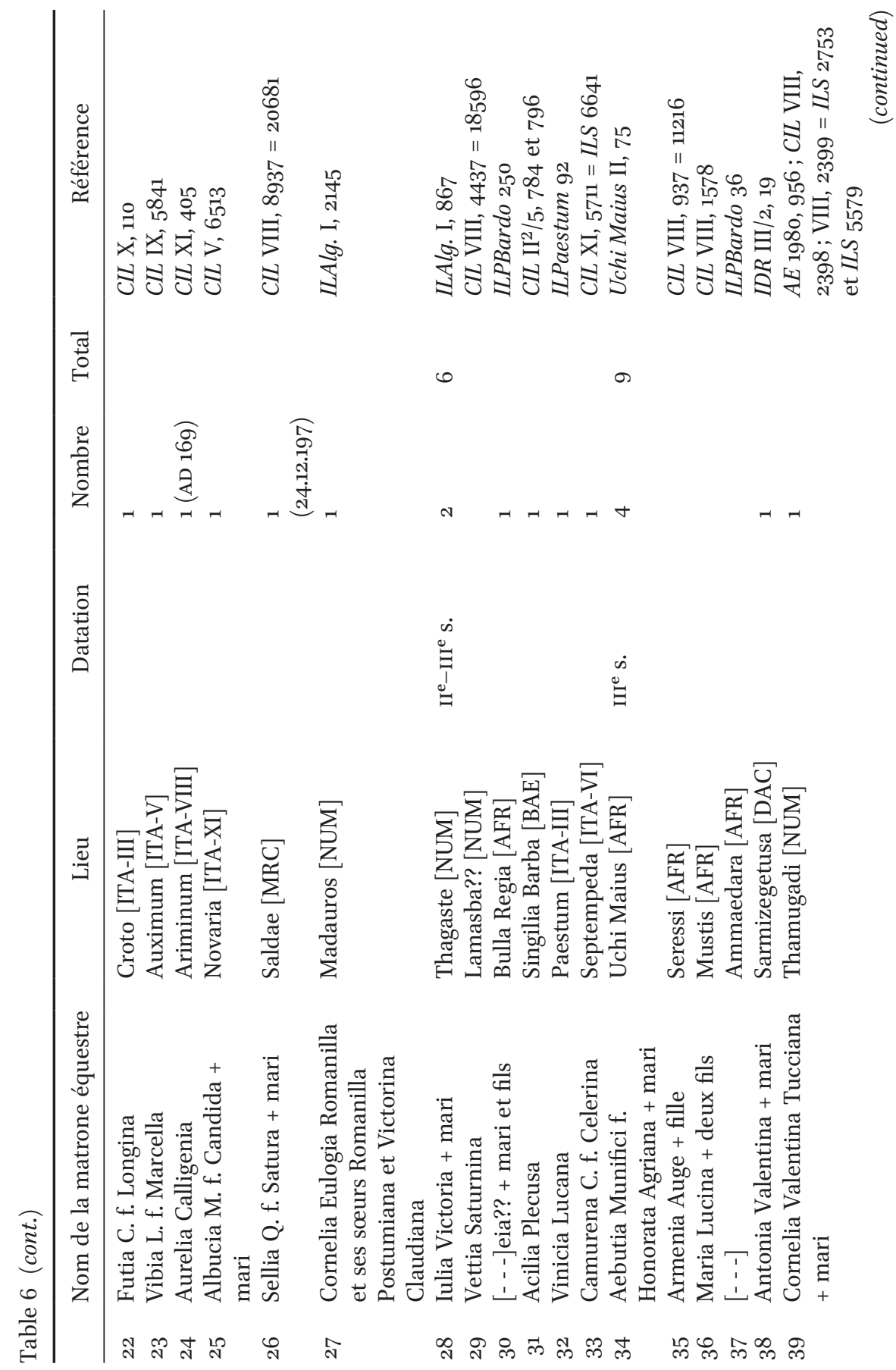




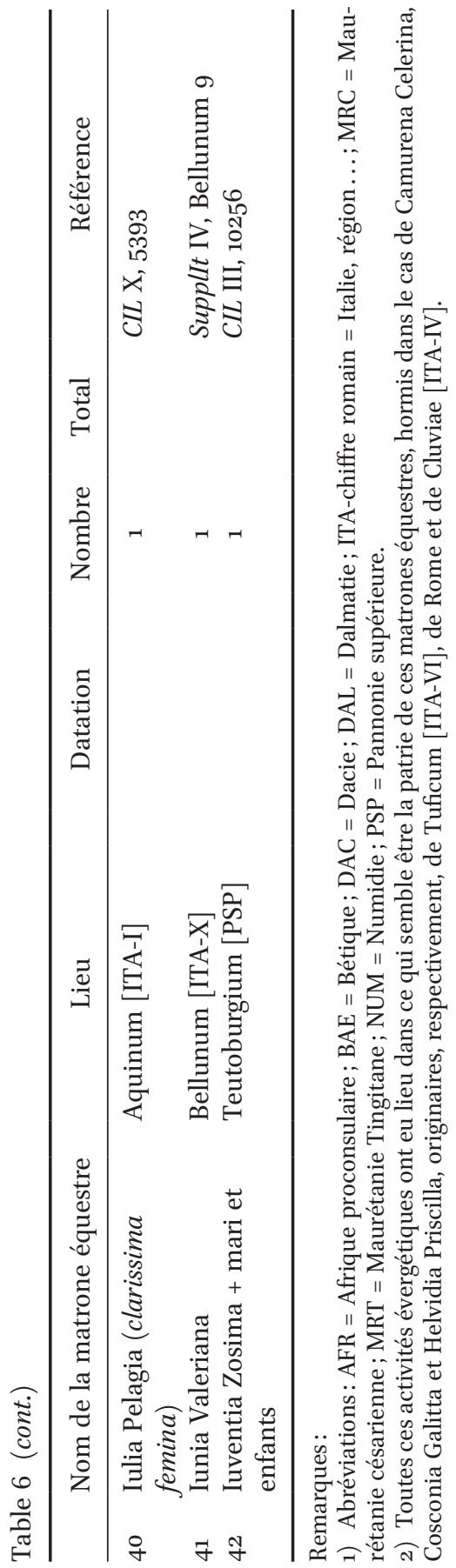

